\title{
George Sand-Victor Hugo, Correspondance croisée
}

\section{Annarosa Poli}

\section{Q OpenEdition}

\section{Journals}

\section{Edizione digitale}

URL: http://journals.openedition.org/studifrancesi/34916

DOI: 10.4000/studifrancesi.34916

ISSN: 2421-5856

\section{Editore}

Rosenberg \& Sellier

\section{Edizione cartacea}

Data di pubblicazione: 1 novembre 2005

Paginazione: 432-433

ISSN: 0039-2944

\section{Notizia bibliografica digitale}

Annarosa Poli, «George Sand-Victor Hugo, Correspondance croisée», Studi Francesi [Online], 146 (XLIX I II) | 2005, online dal 30 novembre 2015, consultato il 19 avril 2021. URL: http://

journals.openedition.org/studifrancesi/34916 ; DOI: https://doi.org/10.4000/studifrancesi.34916

Questo documento è stato generato automaticamente il 19 avril 2021.

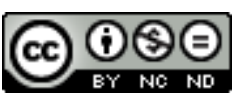

Studi Francesi è distribuita con Licenza Creative Commons Attribuzione - Non commerciale - Non opere derivate 4.0 Internazionale. 


\title{
George Sand-Victor Hugo, Correspondance croisée
}

\author{
Annarosa Poli
}

\section{NOTIZIA}

GEORGE SAND-VICTOR HUGO, Correspondance croisée, lettres réunies et présentées par DANIELLE BAHIAOUI, Nîmes, H. B. Éditions, 2004, pp. 140.

1 Questo volumetto che comprende una Introduzione e il testo di quarantatre lettere scambiate tra Sand e Hugo sarebbe stato di una grande utilità se fossero state indicate le varie edizioni da cui sono state tratte, i volumi e le pagine secondo il normale metodo scientifico.

Nella parte introduttiva, si fa cenno alla Corrispondenza della romanziera curata da Georges Lubin, ma nella seconda, non vi è alcun riferimento preciso a questa edizione né alle diverse edizioni della Corrispondenza di Victor Hugo (V. Hugo, Correspondance, Paris, Calmann Lévy, 1998; Cuvres complètes, éd. J. Massin, Paris, Club français du livre, 1967-70; Euvres complètes, éd. J. Seebacher, assisté de Guy Rosa, Paris, Laffont, 1985-90) incomplete queste ultime in confronto all'edizione magistrale di Georges Lubin. Purtroppo la raccolta della Corrispondenza inizia con una lettera di Hugo del 4 agosto 1864, falsamente datata 4 agosto 1855 tratta dalla edizione C. Lévy della Corrispondenza di Victor Hugo. I rapporti tra George Sand e Victor Hugo sono stati molto trascurati dalla critica, e c'è da chiedersi il perché, un quesito che Mme Bahiaoui non si pone. Avrebbe forse potuto esserle utile la lettura del mio articolo Victor Hugo et George Sand, apparso su «Studi francesi» ( $n^{\circ} 140$, maggio-agosto 2003, p. 330-352).

Molte delle loro lettere sono state pubblicate in articoli o in opere varie, sono stati citati alcuni del feuilletons di lei dedicati alle opere di lui; non esistono contributi del poeta sulla produzione sandiana se non nelle lettere e solo rari cenni del suo interesse per la romanziera prima del 1856. Per conseguenza, si sono tratte conclusioni affrettate sul loro rapporto, ma anche di tutti questi particolari non c'è nessuna notizia da parte 
di Mme Bahiaoui. L'unico studioso che ha dato un quadro verosimile della relazione epistolare è stato Georges Lubin, commentando, nella sua edizione della Correspondance di George Sand le numerose lettere della romanziera al poeta.

4 Da segnalare un breve, ma interessante articolo di K. Biermann, «George Sand et Victor Hugo. Deux visions "parallèles» de la révolution», in Le Siècle de George Sand, textes réunis par A. Powell (Amsterdam-Atlanta, Rodopi, 1998, pp. 29-33) anche questo non citato dall'autrice L'atteggiamento di George Sand verso Victor Hugo era sempre stato in bilico tra l'ammirazione e una critica severa, quasi temesse di lasciarsi trascinare dall'entusiasmo verso colui che nel 1831 la giovane Aurore Dupin definiva «Le plus bavard des poètes sublimes». George è ammirata dalla poesia di Hugo, ma ha delle riserve sulla sua prosa; del suo teatro mette in primo piano Lucrèce Borgia, che tanto l'aveva colpita nel 1833 quando aveva assistito alla rappresentazione. Negli anni Trenta e Quaranta influenzata anche dagli amici letterati che frequentava, persiste nei giudizi negativi. Assiste alla cerimonia di Réception di Sainte-Beuve all'Académie française e, dopo aver ascoltato il discorso di Victor Hugo, scrive un articolo sulla «Réforme» del 2 marzo 1845 in cui il poeta non viene certo risparmiato per la mancanza di un sincero impegno politico e sociale: «Il croit à la puissance de la phrase, à la régénération sociale par la métaphore».

5 Dopo la pubblicazione di Les Châtiments (1853), Sand ancora una volta entra in polemica, a causa del biasimo di lui verso quegli esiliati politici che cercavano di entrare in Francia e per i quali si era tanto adoperata. Quando nel 1856 appare l'opera Les Contemplations, la scrittrice comprende che vi è finalmente una partecipazione profonda dell'anima del poeta nel poema dedicato alla tragica morte della figlia Léopoldine e ne è sconvolta anche per il ricordo della morte recente della sua nipotina Nini. C'è una certa esitazione nella scrittrice a rivolgersi a Hugo e lo farà scrivendo a sua moglie Adèle, la stessa esitazione del poeta che le risponderà indirettamente, tramite Hetzel. Il 5 giugno 1856 inizia così la corrispondenza tra i due (la prima lettera di lui è del 15 giugno $1856 \mathrm{e}$ quella di lei del 21). Segue l'articolo di lei sulle Contemplations apparso sulla «Presse», si moltiplicano gli inviti a Guernesey (che Sand non accetterà mai) e le espressioni calorose di consenso di lui per La Daniella, Les Beaux Messieurs de Bois-Doré. Il 1859 vede i due di nuovo in contrasto per ragioni politiche, ma i rapporti riprenderanno in occasione della Légende des siècles. A proposito del Misérables, nel 1862 si intreccia fra i due scrittori un dialogo di grande interesse perché George vorrebbe vedere lo scrittore più anticlericale, ma questo romanzo finisce per entusiasmarla.

6 La romanziera continua a dedicare articoli a opere del poeta nel 1863 e nel 1864. Nella Corrispondenza si susseguono pagine affettuose in occasione di avvenimenti familiari (la morte del nipotino della Sand la nascita della piccola Aurore) e anche espressioni di grande consenso da parte di Hugo per le opere che la romanziera va pubblicando. Riaffiorano le diffidenze verso l'uomo nel 1871, al tempo della Comune, ma Sand è entusiasta de L'Année terrible che considera il capolavoro del poeta. Una nuova edizione del romanzo Valentine, nel 1875, viene dedicata a Hugo che così si rivolge alla scrittrice: «comme créatrice de chefs-d'œuvre, vous êtes la première de toutes le femmes». E l'anno dopo, in occasione della sua morte, Hugo scriverà il famoso discorso di commemorazione che si conclude con la frase: «Je pleure une morte et je salue une immortelle».

7 Un rapporto tormentato dunque, che va sempre più rafforzandosi con il tempo e con la metamorfosi sociale e spirituale del poeta al quale George Sand dichiara sempre di 
sentirsi inferiore. Vista la ricchezza di testimonianze di questa Corrispondenza, spiace la lacunosa e imprecisa presentazione da parte della curatrice dell'edizione di queste lettere. 